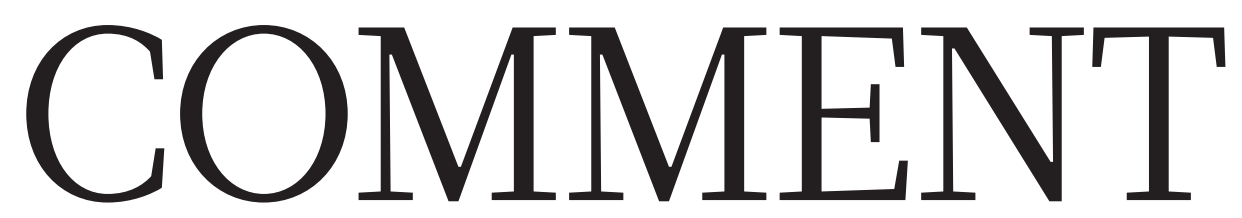

EVoLUTION How mechanisms of fate and chance affect adaptation $\mathbf{p . 1 5 6}$
PALAEOBIOLOGY Why access to samples of ancient DNA must be regulated $\mathbf{p . 1 5 8}$
TAXONOMY Species boundaries should be defined more stably p.158
CRYPTOGOVERNANCE USe the blockchain to trade energy securely $\mathbf{p} .158$

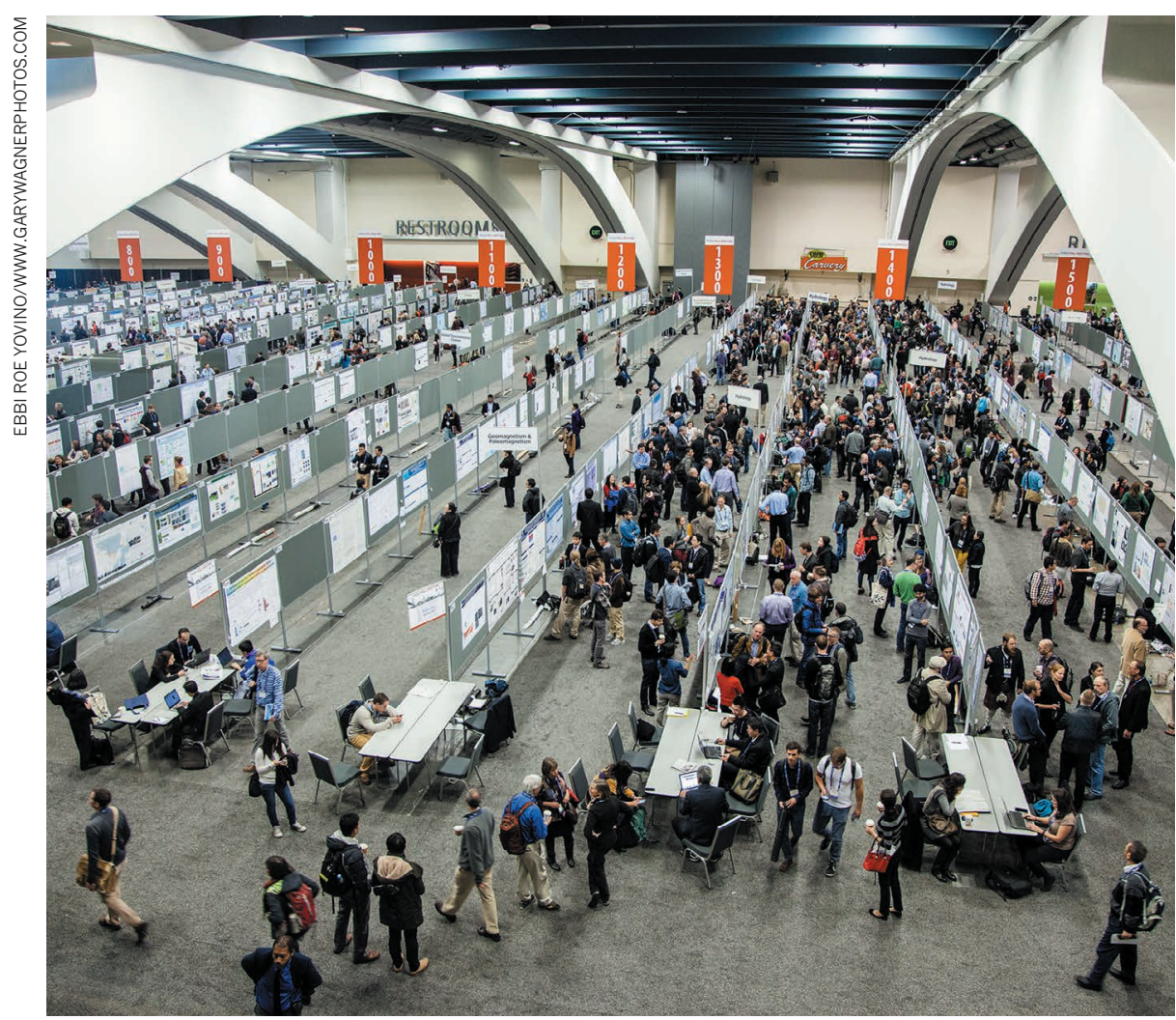

Poster sessions at conferences are often where early research gets its first airing.

\section{Shake up conferences}

\section{Emojis, smartphone technologies and revamped guidelines would boost transparency at scientific meetings, say Shai D. Silberberg and colleagues.}

$\mathrm{M}$ uch ink has been spilt about what journals, funders and research institutions should be doing to promote rigour, reproducibility and better reporting. The role of scientific conferences, where much work gets its first airing, has gone largely unmentioned. Earlier this year, this group of authors, together with other conference organizers and representatives from the US National Institute of Neurological Disorders and Stroke, met to hash out what could be done to improve transparency at meetings. Small pilot studies are already under way.

Attending, presenting and mingling at conferences is crucial for researchers studying topics from astrophysics to zoology. These meetings take many forms. The Federation of American Societies for Experimental Biology (FASEB, with which some of us are associated) hosts more than 30 small research conferences a year, collectively bringing together more than 4,000 attendees. By contrast, the annual meeting of the Society for Neuroscience regularly draws more than 28,000 people.

Conferences have served as crucial hubs for scientific communication for at least four centuries. They provide an essential platform that facilitates collaboration and disseminates information, and they enable researchers to gain feedback on early-stage work. They also train scientists and set standards for quality.

And yet the staples of scientific conferences - presentations and poster sessions can provide only snapshots of ongoing work. This is exacerbated by the complexity of scientific technologies, richness of acquired data and sophistication of data-analysis methods, all of which are ever-growing. As a result, attendees can find it difficult to evaluate presented results and interpret the findings. This hinders their ability to give feedback to colleagues and to decide how to incorporate findings into their own work.

We suggest that a few straightforward strategies could yield vast improvements.

Rewrite presentation and poster guidelines to promote transparency. Instructions for conference participants focus on formats and logistics. For example, at the American Society of Clinical Oncology (ASCO) annual meeting, instructions state that slides for oral presentations must be in 16:9 widescreen format, and presenters are required to declare whether their research was federally funded. Posters must be no larger than 120 centimetres by 240 centimetres and in landscape orientation. Bold, readable fonts are encouraged.

Formatting instructions like these are important, but so are guidelines that promote transparency. Meeting participants should be encouraged to present relevant information regarding the research question and its rationale, whether an experiment is exploratory or confirmatory, what measures were taken to reduce bias, and which controls were used. They should communicate whether the question was approached using different techniques or frameworks, and include sufficient raw data for others to judge the quality of experiments. These items could be compiled as a checklist at the time of abstract submission, even if they are too burdensome to include in the presentation itself. 
> Develop 'rigour emojis'. Emojis - simple picture symbols that can be quickly understood - could be added to slides and poster presentations to convey complicated information efficiently (see 'Delight them with data'). For example, one could use a cartoon of a blindfolded eye to show that experimental treatments were kept hidden as results were collected and data analysed; a single playing die to show that samples were randomized into comparison groups; or a checkmark to indicate that the study is confirmatory rather than exploratory. A computer power button or large $N$ symbol could indicate that appropriate sample sizes were calculated before conducting experiments, thus ensuring rigour.

FASEB is piloting the use of a handful of rigour emojis at its Science Research Conferences (SRCs). Participation is voluntary. The extent to which presenters adopt the practice will help to gauge whether the community finds the emojis helpful, whether the symbols have an impact on how the audience interprets the data, and whether they can be integrated into presentations without disrupting the flow of the sessions.

Symbols could be created and used in accordance with what each community judges to be most important. For instance, FASEB has suggested using a stamp on posters or slides saying 'littermate controls' to show that mice from the same litters were split into treatment and non-treatment groups. A clinical-trials conference, by contrast, might use symbols to indicate that diverse populations were included in treatment studies or that trials used common data elements that standardize the collection of data.

Mobilize technology. Technology could be harnessed more efficiently at conferences to make more information available. For example, electronic posters allow viewers to zoom in on experimental details. Perhaps they will be implemented more widely once they become more affordable and practical. Conventional posters could include Quick Response (QR) barcodes, as suggested by the ASCO. These would allow attendees to use their smartphones to view conference or laboratory websites for additional information, such as a copy of the poster, papers supporting the scientific premise of the study or in-depth experimental details and data summaries for each figure.

There should also be ways to enable attendees to use social-media platforms such as Twitter to discuss experimental details and allow presenters to respond without excessive burden. These conversations, if hosted by the conference organizers, could be curated so that they are compiled and moderated in the way that is most helpful to the community.

\section{DELIGHT THEM WITH DATA}

Two charts of the same data reveal ways to enhance transparency. (A) shows only an overview of the data, but (B) includes much more detail.

\section{A Little transparency}

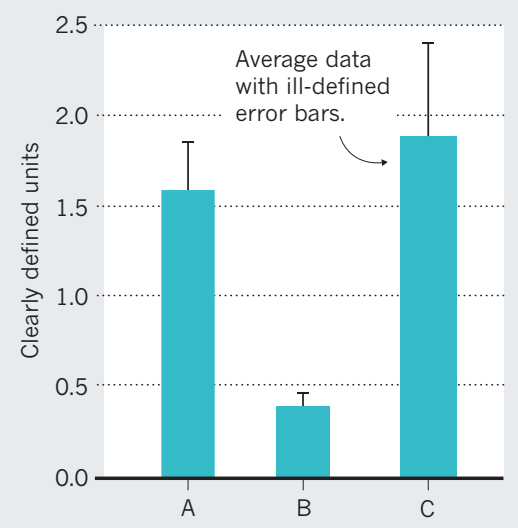

B More transparency

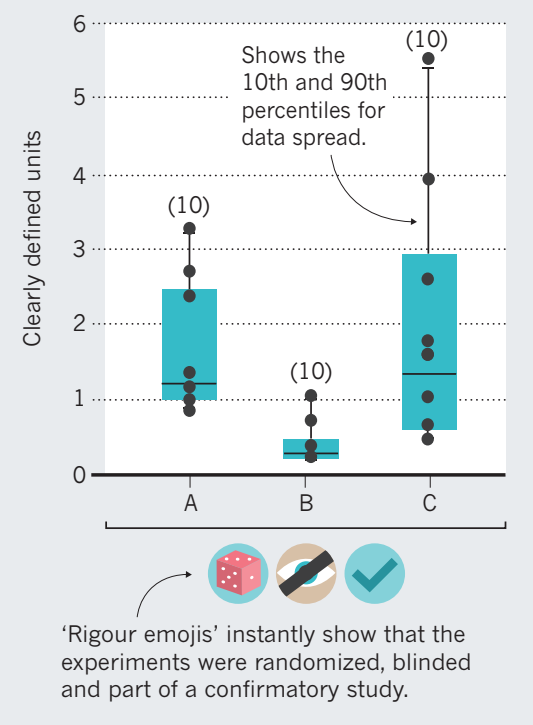

Lead by example. Clinical researchers generally disclose commercial interests and other potential conflicts at the beginning of their presentations. Adding transparency elements to talks and posters could also become commonplace. The ASCO promotes the use of a symbol or badge to disclose federal funding, and rigour emojis could similarly be explained at the beginning of a talk.

If senior scientists and rising stars adopt these symbols and other transparency practices, they could start a lasting trend. Trainees will learn that rigour and transparency are valued by the scientific community and will start to incorporate them into their own presentations. In this way, the next generation of scientists can shape the culture of rigour and transparency.

Furthermore, training courses could be run during conferences to provide tips and tricks on incorporating different elements of transparency into various presentation formats. If these courses were led, promoted and attended by senior scientists as well as junior scientists, this could quickly increase the popularity of such elements.

Pilot, assess and tweak. Multiple methods for increasing transparency in presentations will need to be tested so that the most effective ones can be adopted depending on the scientific field, the conference format or the needs of individual scientists. Simply asking about certain elements of rigour will probably increase attention to them.

FASEB conference organizers are currently surveying audience members and presenters at their SRCs about their reactions to and suggestions for different transparency efforts. As part of these pilot studies, FASEB will also assess how much effort is required to implement these changes. New practices will have little impact if they are not sustainable.

There may be barriers to adoption of such practices, including increased time requirements, resistance to change, regulatory burdens or pressure to present only studies that are mature. Conferences are crucial for communication about work that is in progress, hence greater transparency should be encouraged and embraced by all attendees. Ongoing efforts in this area will help to inform the development of practices that apply across many disciplines and meeting formats.

These strategies are suggestions, and we don't want to overreach. Strict rules are neither feasible nor desirable. Conferences have diverse structures, varied presentation formats and many field-specific idiosyncrasies.

Meaningful change is possible. From 2013, journals such as Nature introduced checklists to improve reporting in research papers. In 2016, the US National Institutes of Health added guidelines for grant applications to help reviewers and applicants to address rigour. Alongside grants and publications, organized meetings are crucial for scientific progress. As scientists, we should be doing experiments to determine how conferences can best advance science.

Shai D. Silberberg is director of research quality, Devon C. Crawford is a health programme specialist, Robert Finkelstein is director of the Division of Extramural Activities and Walter J. Koroshetz is director at the National Institute for Neurological Disorders and Stroke in Bethesda, Maryland, USA. Robert D. Blank is chair of the SRC advisory committee, Hudson $\mathbf{H}$. Freeze is past president, Howard H. Garrison is deputy executive director for policy and Yvette R. Seger is director of science policy at the Federation of American Societies for Experimental Biology in Bethesda. e-mail:silberbs@ninds.nih.gov 